\title{
Design and Fabrication of Vascular Network for Muscle Tissue
}

\section{Engineering}

\author{
Guirong Dong ${ }^{123}$, Qin Lian ${ }^{3 a}$, Laixia Yang ${ }^{1}$,Wei Mao ${ }^{3}$, Shiyang Liu ${ }^{3}$, Chao Xu ${ }^{1}$ \\ ${ }^{1}$ School of Mechanical Engineering, Xi'an University of Sicense and Technology, Xi'an 710054,
} China.

${ }^{2}$ Faculty of Higher Vacational and Technical Education, Xi'an University of Technology, Xi'an 710082, China.

${ }^{3}$ State Key Laboratory for Manufacturing Systems Engineering, Xi'an Jiaotong University, Xi'an 710049, China.

a E-mail address: 38296180@qq.com. (Q. Lian).

Keywords: Vascular network, Gelatin hydrogel, Muscle tissue engineering

Abstract: Function loss of large skeletal muscle is an urgent problem in the clinic. Although the artificial microfluidic network encapsulated in thick tissue-engineered constructs has a great promise, it is still difficult to develop an efficient microfluidic design to fit the requirements of the bionic vascular network for tissue engineering application. In this study, a seriers of biomimetic principles was outlined and a vascular network for muscle tissue engineering was designed following these principles. CFD analysis indicated that the velocity error of fabricated model was controlled within $10 \%$ in the different pressures, the velocity curve of designed model and fabricated model are better matched when the inlet pressure is $6 \mathrm{mmHg}$. Therefore, the future work will focus on the in vitro tests with this inlet parameter to verificate and optimize the vascular networks and validating their ability to support myoblast for clinical use.

\section{Introduction}

Various reasons cause the defection and the function loss of large volume of skeletal muscle, such as congenital defect, injury, tumor, primary myopathy and metabolic diseases.The traditional treatment method is totransplant the myocutaneous flap to the defection area, however, there still are few challenges such as the limitation of muscle sources, the damage of donators and immunological rejection etc [1].

In the past few years,some scientists used the myoblast transplantation to promote the muscle fiber regeneration and treat genetic myopathy, but there are some problems remaining such as uneven distribution of myoblasts in the human body, low survival rate and immune rejection[2]. Whereas the skeletal muscle tissue engineering is developing fast nowadays, which will very likely to bring over a bright future in the field of the structure and function repairmen [3].There are abundant vessels existing in the muscle, the muscle cell mass exceeds $1 \mathrm{~mm}^{3}$ would die without vessels growing. The microfluidic networks could initially function as the fluidic pathways for effective mass transport inside the constructs [4].

Recently, there are significant efforts in designing and fabricating muscle scaffolds for the 
microfluidic network, the materials of scaffolds including collagen, gelatin, alginate, PLGA and PEG hydrogels, even the silica gel [5-6]. In field of tissue engineering, there are many methods to fabricate the muscle tissue engineering scaffolds, such as molding, photoresist self-assembly method, electrostatic spinning, etc. Through cultivating the scaffolds which are seeded cells to stimulate the cells differentiation for implementing structure and part of the muscle function [7-9].

The function of the microfludic network is very important which provides the exchange of nutrients and space for cell production and migration. However, In some previous work, there is lack of microfludic in the scaffolds, merely random pores which is caused by using freeze-drying and phase separation methods [10,11]. In some other studies, Parallel arrangement of the microarchitectures such as fibers, grooves, and pits on the scaffolds are created by chemical or topographic patterning, although this microstructure can promote cell alignment and differentiation, they could not fit the requirements of the bionic [12-13]. Designing a scaffold which have bionic microfluidic networks is still a challenge.

In this work, we present a facile approach to fabricate branched network with square cross-sections in the gelatin hydrogels based on the bionic design principle of blood vessels and CFD analysis, muscle scaffold model with bionic microfluidic network is established. Stereolithography (SL), a kind of 3D printing technique was used to fabricate resin mold with semi-square microchannel, which was transferred to PDMS molds for monolayer microfluidic hydrogel replication. Two hydrogel layers were assembled to form a square channel. The channel morphology was evaluated by optical microscopy. CFD analysis was used to recorded the course of pressure drop and velocity change of the designed model and fabricated models to provide the design theory for the further cell culture in vitro.

\section{Materials and methods}

Biomimetic design principles. To increase the nutrition of transmission to fabricate large size scaffolds, the key point is to design a bionic microfluidic network in the scaffolds which is advantageous to the microfludic internal blood flow uniformity, besides, it is easy to realize blood perfusion. Vascular network design principles are shown in Table 1.

Table 1Biomimetic Vascular Network Design Principles

\begin{tabular}{lll}
\hline Principles & Rules \\
\hline 1 & $\begin{array}{l}\text { Defined relationship } \\
\text { between parent } \\
\text { and daughter diameters }\end{array}$ & $\begin{array}{l}\text { Murray's Law [14] } \\
r_{0}^{3}=\mathbf{r}_{1}^{3}+r_{2}^{3}\end{array}$ \\
$\begin{array}{l}\text { Physiologic branching } \\
\text { angle }\end{array}$ & $90^{0}[15]$ or $75^{0}[16]$ \\
3 & Biomimetic vessel length & $\begin{array}{l}\text { Avoid long, small-diameter channels to minimize } \\
\text { platelet activation and thrombus formation [17] }\end{array}$ \\
4 & $\begin{array}{l}\text { Aspect ratio of vascular } \\
\text { channels }\end{array}$ & $\begin{array}{l}\text { Biomimetic radial design with physiologic shear } \\
\text { stress and 1:1 aspect ratio of all channels [18] }\end{array}$ \\
5 & Venous scaling & $\begin{array}{l}\text { Venous lumen area approximately are 10\% larger } \\
\text { than artery lumen area [19] }\end{array}$ \\
6 & $\begin{array}{l}\text { Blood vessels bifurcations divisions of blood vessels in two bifurcations is } \\
\text { design }\end{array}$ & $\begin{array}{l}\text { beneficial to the uniform flow of blood in the lumen } \\
{[20]}\end{array}$ \\
\hline
\end{tabular}

In equations (1), $\mathrm{r} 0$ is the radius of the parent vessel diameter, $\mathrm{r} 1$ and $\mathrm{r} 2$ are the radii of the daughter vessel diameters. 
Vascular network design. Scaffold model was designed based on the design principles shown in Table 1, UG software was used to design a 40 layers cylinder assembly scaffolds was drawn (Fig. 1a), the top layer is the splitter plate. Fig. $1 \mathrm{~b}$ shows the disc-shaped monolayer which is composed of uniform distribution of six groups of microfludic, the one-sixth microfludic cell sketched from artery to vein was shown in Fig. 1c, the size of different levels and bifurcation angle have been listed in table 2.

a)

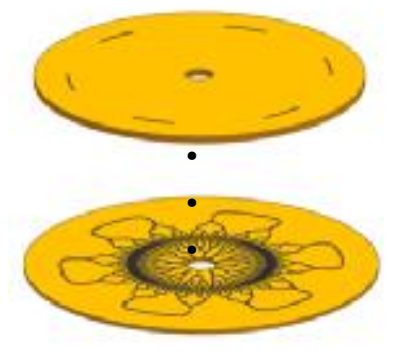

b)

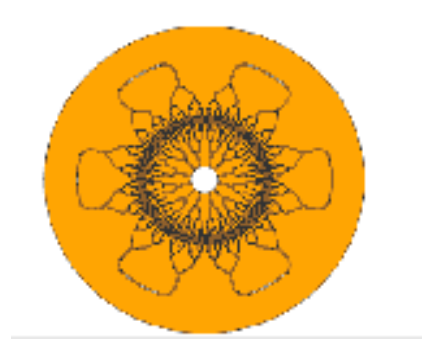

c)

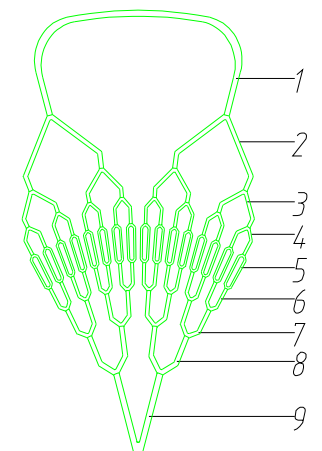

Fig. 1 radial branching vascular network model. a) Assembly diagram of 40 layers scaffolds, b) Exploded view of single-layer, c) The structure of one-sixth vascular network.

Table 2 Vascular Network Dimensions, length and angle for Each Generation.

\begin{tabular}{lcccc}
\hline No & $\begin{array}{c}\text { Vascular network } \\
\text { channel }\end{array}$ & $\begin{array}{c}\text { Channel } \\
\text { Diameter } \\
(\mathrm{mm})\end{array}$ & $\begin{array}{c}\text { Channel } \\
\text { length } \\
(\mathrm{mm})\end{array}$ & $\begin{array}{c}\text { Angle } \\
\left(^{\circ}\right)\end{array}$ \\
\hline 1 & Inlet channels & 673 & 10.09 & 32 \\
2 & 2nd-generation inlet & 534 & 5.74 & 80 \\
3 & 3rd-generation inlet & 424 & 4.44 & 80 \\
4 & 4th-generation inlet & 334 & 3.44 & 80 \\
5 & Smallest channel & 267 & 6.8 & 90 \\
6 & 1st-generation outlet & 364 & 3.44 & 90 \\
7 & 2nd-generation outlet & 458 & 4.44 & 95 \\
8 & 3rd-generation outlet & 576 & 5.74 & 90 \\
9 & 4th-generation outlet & 727 & 10.09 & 32 \\
\hline
\end{tabular}

CFD parameters. CFD is a powerful tool to simulate fluid flow and optimize flow parameters before manufacturing vascular networks.CFD has been used optimizing the blood flow in other microvascular networks [21].

The radial symmetry of the design enabled the blood flow to be simulated in only one-sixth of the vascular network design. AUTO CAD was used to create the 2D geometric vascular network model, a computational mesh was generated using Gambit software. The cell spacing of simulation mesh is $0.1 \mathrm{~mm}^{2}$. Moreover, the curve of arteries side is defined as the pressure inlet, and the line of vein side is defined as the pressure outlet. Non-slip wall conditions were applied along the internal surface of the microchannels. A K-Epsilon model which is a non-Newtonian blood model was used to analyze the blood viscosity. The CFD analysis was astringed within 200 iterations. The inlet pressure boundary was Set respectively as $0.5,2,4,6,8,10 \mathrm{mmHg}$, the output press boundary was set as $0 \mathrm{mmHg}$.

Materials and manufacture process. After constructing muscle scaffold 3D model with UG based on the 2D geometric vascular network model, stereolithography (SL) was used to fabricate 
the resin mold with the semi-rectangular microchannels, casting Sylgard 184 polydimethylsiloxane (PDMS)to get the mold, and then pouring the Fresh $10 \%(\mathrm{w} / \mathrm{v})$ gelatin solution into the mold to prepare scaffolds, placed at room temperature for $15 \mathrm{~min}$ and then cooled to $4{ }^{\circ} \mathrm{C}$ for $30 \mathrm{~min}$ to induce gelation. The partially crosslinked gelatin hydrogels with semi-square channels were carefully demoulded. Assembling two semi-network layers with the guide of the position holes carefully (Fig.2).

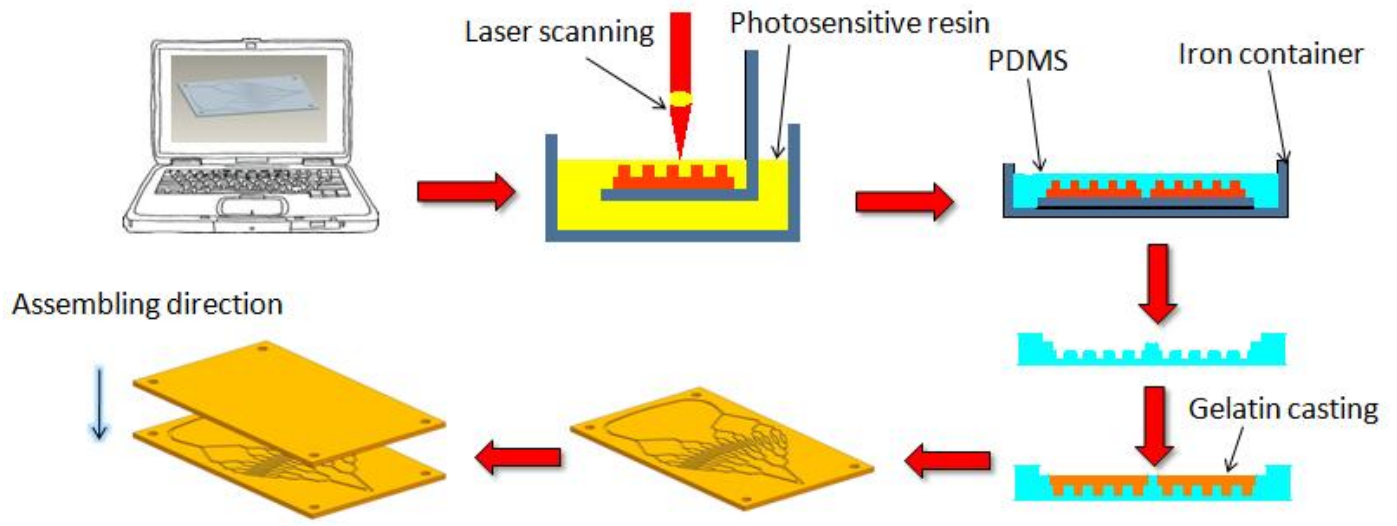

Fig. 2 Schematic of prepare muscle tissue engineering scaffolds with microfluidic networks.

modeling accuracy. To characterize the microchannels in the assembled gelatin hydrogel, the fresh sample was exposed under the optical microscopy (Ti-5, Nikon). To quantify the fabrication accuracy of the micro-channels, six hydrogel samples were totally characterized and the quantitative data was expressed as mean \pm standard deviation. Modeling accuracy according to the law:

$$
s=\frac{A-B}{A} \times 100 \%
$$

In equations (2), $\mathrm{S}$ is the microchannels, forming accuracy, $\mathrm{A}$ is the actual average dimension , $\mathrm{B}$ is the design dimension.

\section{Results}

Modelling accuracy. Fig. 3a shows the microfluidic gelatin hydrogel assembled from two partially gelatin slides. The overall thickness of the assembled hydrogel is about $4 \mathrm{~mm}$. The guided stacking process can accurately position the two semi-square microchannels form the closed microfluidic network. Fig. $3 b-j$ show that the width of the microfluidic channel network characterized at different levels by the optical microscopy.

The width of microfluidic channels was measured from the semi-square images (Fig. 4). It was found that the width of the fabricated channels from level 1 to 9 are $697 \pm 23 \mu \mathrm{m}, 584 \pm 18 \mu \mathrm{m}$, $472 \pm 14 \mu \mathrm{m}, \quad 369 \pm 16 \mu \mathrm{m}, 275 \pm 3 \mu \mathrm{m}, 403 \pm 20 \mu \mathrm{m}, 501 \pm 9 \mu \mathrm{m}, 622 \pm 14 \mu \mathrm{m}, 748 \pm$ $12 \mu \mathrm{m}$, respectively which are corresponding to the forming accuracy of $3.57 \%, 9.4 \%, 11.3 \%$, $10.5 \%, 3 \%, 10.7 \%, 9 \%, 8 \%$ and $3 \%$. 


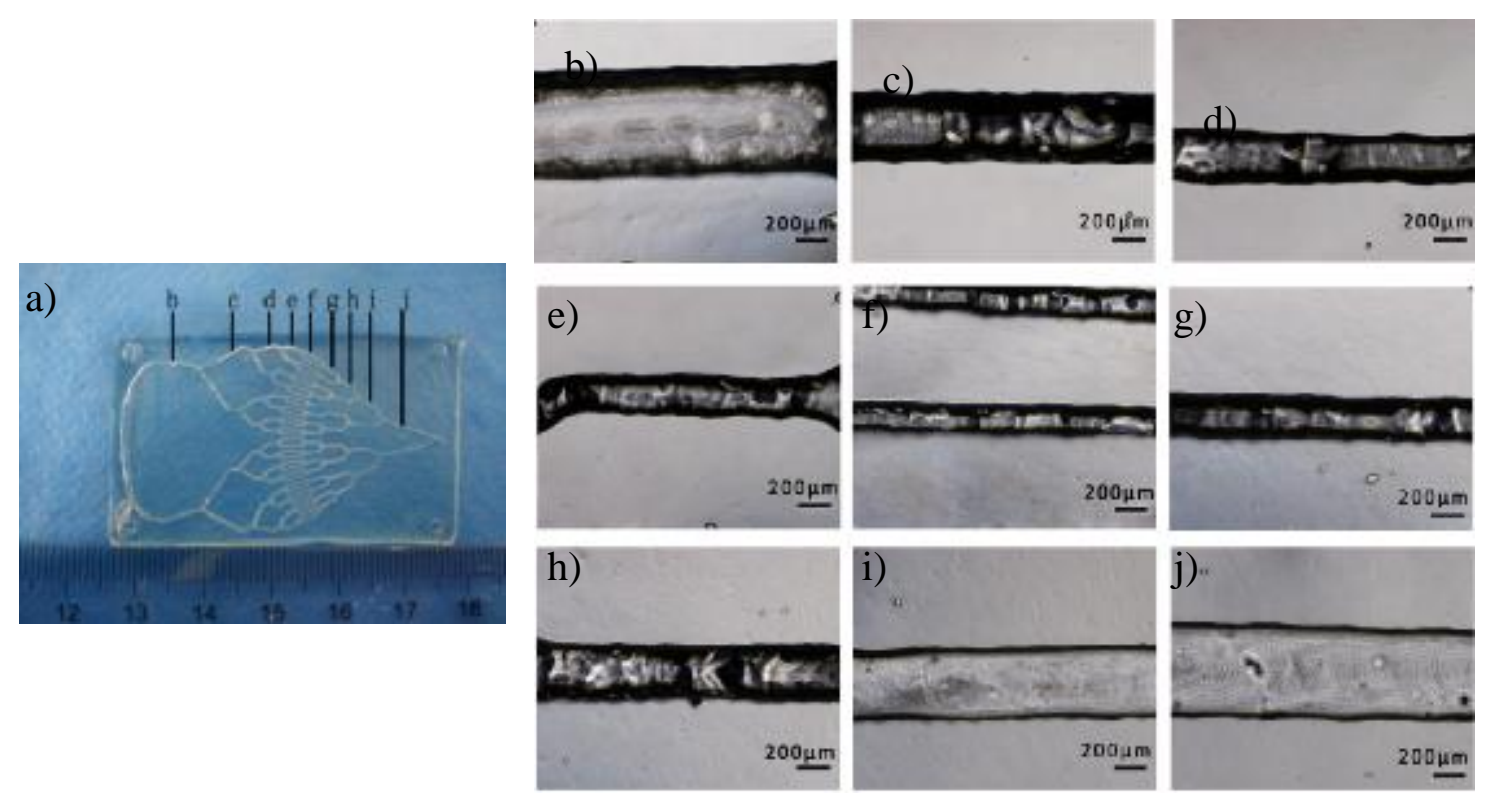

Fig. 3 gelatin hydrogel scaffolds with semi- microchannels. (a) Photograph of the fabricated microfluidic hydrogel, $(b-j)$ Optical microscopy images of hydrogel microchannels at different levels(respectively corresponding 1 to 9 channel).

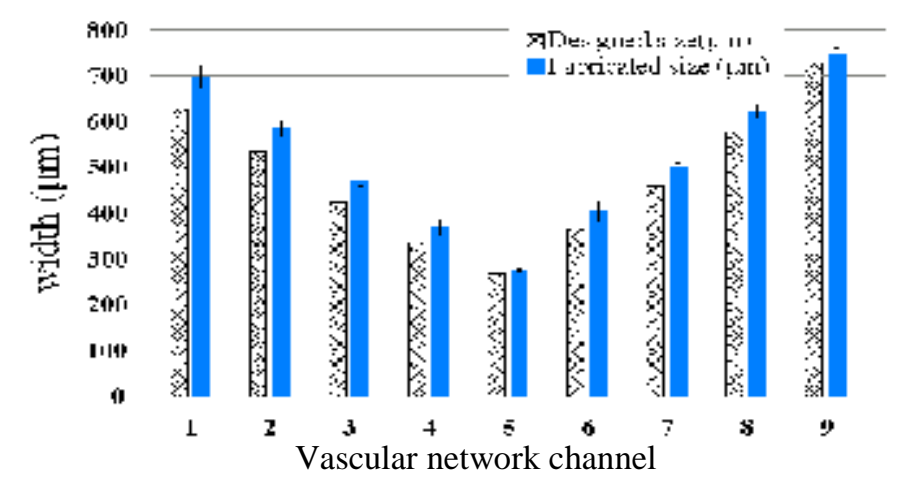

Fig. 4 Graph of design size and forming size respectively to different levels (error bars \pm standard deviation) .

CFD analysis. CFD analysis was employed to estimate the fabrication error on the fluid flow in the microfluidic hydrogels. Fig. 5a shows the pressure distribution inside both models exhibiting negligible difference. Fig. 5b shows the velocity distribution inside both models. The results show that the pressure drop is uniform inside the vessel network, there is more pressure drop occurring in the inlet generations than the outlet generations. Velocity drops with the increasing number of microchannels, nevertheless, the maximum inlet velocity appears at the center of the channels as shown in Fig. $5 \mathrm{c}-\mathrm{h}$. Under the different pressure, maximum velocity error in the center of the inlet channels are respectively $9.4 \%, 8.1 \%, 7.5 \%, 7.1 \%, 7.2 \%, 7.1 \%$. 
a)

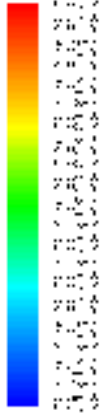

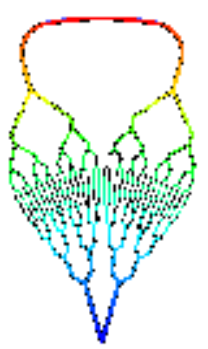

$\triangle$ c)

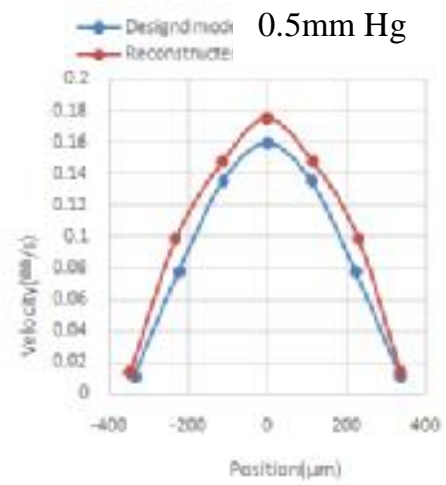

f)

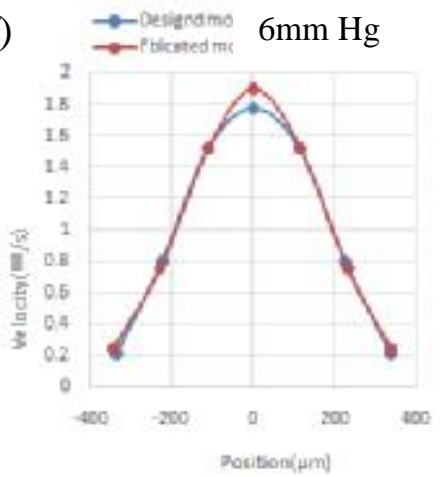

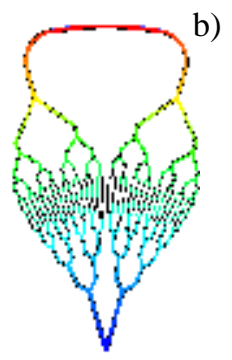

b)
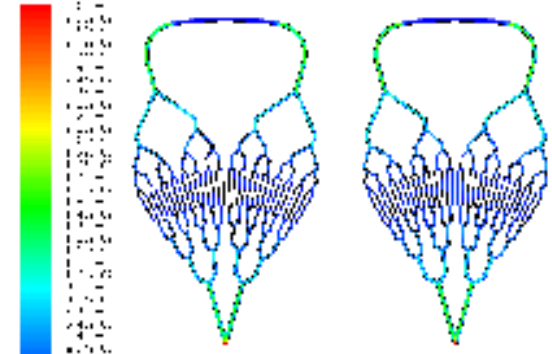

$\Delta$ d)

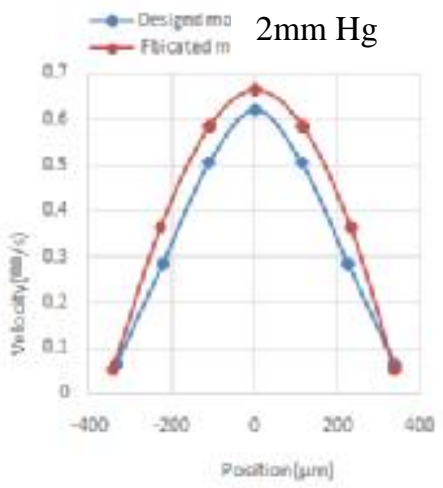

g)

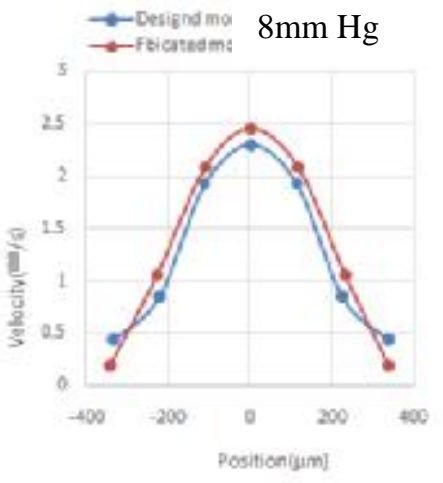

e)

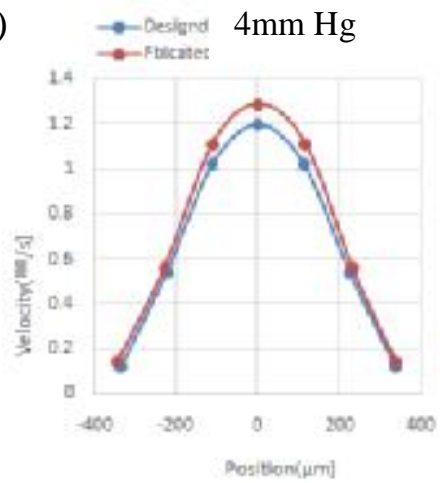

h)

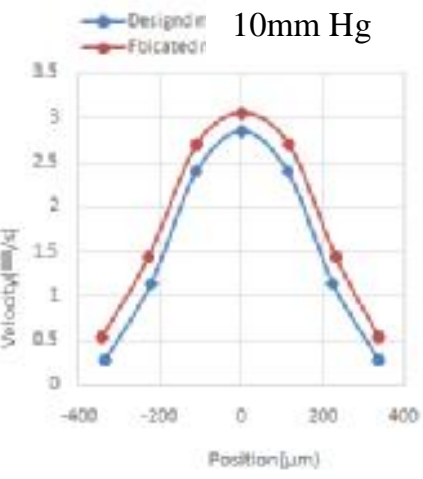

Fig. 4 CFD analysis of fluid flow in designed and fabricated models. (a) Pressure distribution in designed model $(\boldsymbol{\Delta})$ and reconstructed $\operatorname{model}(\triangle)(0.5 \mathrm{mmHg})$, (b) velocity distribution in designed and reconstructed models, $(\mathrm{c}-\mathrm{h})$ velocity quantification in designed and fabricated models under the different pressure in the pressure inlet.

\section{Discussion}

Branched vascular networks are a central component of the scaffolds structure for the large size organ tissue engineering. This work presents a biomimetic design methodology founded on the independently investigated anatomic and physiological principles of the natural blood vessels. Some research on blood vessel sizes [22], the errors of microchannels between the theoretical model and fabricated model are inevitable [20].

As the denatured product of collagen, gelatin is low cost and can be easily modified to improve its processibility and simultaneously maintain its bioactive properties. This study found that the widths of the microchannels in the gelatin scaffold was increased after molding. The forming 
temperature of gelatin at $37^{\circ} \mathrm{C}$ may be the major reason because when the temperature decreases to the room temperature, gelatin scaffolds will have a certain amount of shrinkage after demoulding. The fabricated error is approximate to $3 \%$ when the widths of microchanels are less than $300 \mu \mathrm{m}$ and more than $650 \mu \mathrm{m}$. The modeling accuracy of other sizes was not ideal that the maximum value was $11.3 \%$. To qualify the size change for the time being, the further experiments will be addressed.

To estimate the effect of the biomimetic microchannel on the flow distribution, CFD was chosen to analyze the fluid flow in the design model and fabricated scaffolds. After exerting the same pressure on the both models, the distribution of flow velocity at the entrance shows that fabrication error about 3\% has little influence to the liquid distribution in the microchannels, only the velocity value of fabricated model was slightly faster than the design model, while velocity figures of two different models presented the highest degree of match when the inlet pressure is 6 mmHg. David's experiment proofs when the inlet pressure sets to $6 \mathrm{mmHg}$, velocity of the designed model was closest to in vitro test, the error was even less than 5\% [19]. Microfluidic vascular networks can deliver oxygen and nutrients to ambient cells. However, long channels with very small diameters may cause flow disturbances and possibly platelet activation [23]. In order to optimize the structure of vascular network, the in vitro experiment was essential to performed.

\section{Conclusion}

Microfluidic vascular networks can deliver oxygen and nutrients to the ambient cells, it may be used to develop tissue engineered organs and organ assisted devices. In this work, the scaffold with vascular Network has been designed. CFD analysis demonstrated that the velocity error of fabricated model controlled within $10 \%$ under different pressure, the velocity curve of both models are match well when the inlet pressure is $6 \mathrm{mmHg}$. The future work will focus on the in vitro tests with this inlet parameters to verificate and optimize the vascular networks and validating their ability to support myoblast for clinical use.

\section{Acknowledgments}

This work was supported by the National Natural Science Foundation of China (51375371) and the high-tech projects of China (2015AA020303).

\section{References}

[1]Yang, H. S.; Ieronimakis, N.; Tsui, J. H.; Kim, H. N.; Suh, K. Y.;Reyes, M.; Kim, D. H. Nanopatterned Muscle Cell Patches for Enhanced Myogenesis and Dystrophin Expression in a Mouse Model of Muscular Dystrophy. Biomaterials (2014), 35, 1478-1486.

[2]Peter X. Ma. Biomimetic materials for tissue engineering[J]. Advanced Drug Delivery Reviews, (2013), 60(2): $184-98$.

[3]Chart BP,Leong KW. Scaffolding in tissue engineering: general approaches and tissuespecific considerations[J]. Eur Spine J, (2008), 1 7(Suppl 4): 467-79.

[4] J.S. Miller, K.R. Stevens, M.T. Yang, B.M. Baker, D.H. Nguyen, D.M. Cohen, et al., Rapid casting of patterned vascular networks for perfusable engineered three-dimensional tissues, Nat. Mater. 11 (2012) 768 - 774. 
[5] Hosseini V, Kollmannsberger P, Ahadian S, et al. Fiber-Assisted Molding (FAM) of Surfaces with Tunable Curvature to Guide Cell Alignment and Complex Tissue Architecture[J]. Small, (2014), 10 (23): 4851-4857.

[6] Martin NR, Passey SL, Player DJ, et al. Factors affecting the structure and maturation of human tissue engineered skeletal muscle[J]. Biomaterials, (2013), 34 (23): 5759-5765.

[7] Bajaj P, Rivera JA, Marchwiany D, et al. Graphene-based patterning and differentiation of C2C12 myoblasts[J]. Adv Healthc Mater, (2014), 3 (7): 995-1000.

[8] Takahashi H, Shimizu T, Nakayama M, et al. The use of anisotropic cell sheets to control orientation during the self-organization of 3D muscle tissue[J]. Biomaterials, (2013), 34 (30): 7372-7380.

[9] Cui XF, Gao GF, Qiu YJ. Accelerated myotube formation using bioprinting technology for biosensor applications[J]. Biotechnology Letters, (2013), 35 (3): 315-321.

[10]Chen MC, Sun YC, Chen YH. Electrically conductive nanofibers with highly oriented structures and their potential application in skeletal muscle tissue engineering $[\mathrm{J}]$. Acta Biomater, (2013), 9 (3): 5562-5572.

[11] Ma, P. X.; Zhang, R. Y. Microtubular Architecture of Biodegradable Polymer Scaffolds. J. Biomed. Mater. Res. (2001),56, 469-477.

[12] Yang, H. S.; Ieronimakis, N.; Tsui, J. H.; Kim, H. N.; Suh, K. Y.; Reyes, M.; Kim, D. H. Nanopatterned Muscle Cell Patches for Enhanced Myogenesis and Dystrophin Expression in a

Mouse Model of Muscular Dystrophy. Biomaterials (2014), 35, 1478-1486.

[13] Li, Y.; Huang, G.; Zhang, X.; Wang, L.; Du, Y.; Lu, T. J.; Xu, F. Engineering Cell Alignment in Vitro. Biotechnol. Adv. (2014),32, 347-365.

[14] Jana, S.; Leung, M.; Chang, J.; Zhang, M. Effect of Nano- and Micro-Scale Topological Features on Alignment of Muscle Cells and Commitment of Myogenic Differentiation. Biofabrication (2014), 6, 035012-1_12.

[15] Zan, X.; Feng, S.; Balizan, E.; Lin, Y.; Wang, Q. Facile Method for Large Scale Alignment of One Dimensional Nanoparticles and Control over Myoblast Orientation and Differentiation. ACS Nano (2013), 7, 8385-8396.

[16] Murray, C.D. The physiological principle of minimum work: I. The vascular system and the cost of blood volume. ProcNatl Acad Sci U S A(1926), 12, 207.

[17]Zamir, M. Optimality principles in arterial branching. J TheorBiol(1976) , 62, 227.

[18]Huang Z, Li X, Martins-Green M, et al. Microfabrication of cylindrical microfluidic channel networks for microvascular research[J]. Biomed Microdevices, (2012), 14 (5): 873-883.

[19] Milnor, W. Hemodynamics, 2nd edition. Baltimore: Williams\& Wilkins, (1989).

[20]David M. Principles of Biomimetic Vascular Network Design Applied to a Tissue-Engineered Liver Scaffold TISSUE ENGINEERING: Part A(2010), Volume 16, Number 5.

[21] Jiankang HeFabrication of circular microfluidic network in enzymatically-crosslinked gelatin hydrogelMaterials Science and Engineering C (2016), 53 - 60 
[22] Park, J., Li, Y., Berthiaume, F., Toner, M., Yarmush, M.L., and Tilles, A.W. Radial flow hepatocyte bioreactor using stacked microfabricated grooved substrates. (2008)Biotechnol Bioeng $99,455$.

[23]Player DJ, Martin NR, Passey SL, et al. Acute mechanical overload increases IGF-I and MMP-9 mRNA in 3D tissue-engineered skeletal muscle[J]. Biotechnol Lett, (2014), 36 (5): 1113-1124. 